J. Appl. Cryst. (1974). 7, 147

\title{
Finite Slit-Height Correction for the Study of Systematic Deviations from Porod's Law
}

\author{
By W. Ruland \\ Universität Marburg, Fachbereich Physikalische Chemie, Bereich Polymere, Lahnberge, Gebäude H, \\ Germany $(B R D)$
}

\begin{abstract}
The evaluation of the small-angle scattering of two-phase systems is greatly facilitated if Porod's law can be observed in the outer angular region. A finite width of the phase boundary as well as density fluctuations within the phases produce systematic deviations from Porod's law which can be determined and eliminated by $s^{4} I-s^{n}$ plots in the case of pin-hole collimation and $s^{3} J-s^{n}$ plots in the case of an 'infinite' slit-height. Unfortunately, the study of the systematic deviations very often necessitates measurements in angular regions for which the assumption of an infinite slit height is no longer valid. However, if the lower limit of the validity of Porod's law is equal or smaller than half of the extent of the constant inner part of the slit function, a complete evaluation of the scattering is still possible. The method consists in replacing the factors $s^{n}$ in the above-mentioned plots by appropriate expressions deduced from the slit function. In a first step, the parameters related to the slope and the intercept of this plot are obtained which, in further steps, are used to correct the integral values which are needed to compute the invariant and the length of coherence. If the slit function can be approximated by a trapezium, all correction functions can be given in analytical form. The method is applied to some earlier measurements on glassy carbons and it is shown that neglecting the effect of a finite slit height leads primarily to underestimating the values for the density fluctuations whereas the pore-size parameters and the porosity values are much less affected.
\end{abstract}

J. Appl. Cryst. (1974). 7, 147

\section{A New Iterative Method for Collimation Correction in Small-Angle Scattering}

\author{
By Otto Glatter \\ Institut für Physikalische Chemie, Universität Graz, Heinrichstrasse 28, A-8010 Graz, Austria and Institut \\ für Röntgenfeinstrukturforschung, Graz, Steyrergasse, Austria
}

(Received 2 January 1973; accepted 16 October 1973)

\begin{abstract}
A new iterative procedure for desmearing small-angle scattering data including the three types of resolution errors and arbitrary weighting functions is presented. The statistical errors of the data are taken into account by a 'weighted least-squares' approximation, i.e. the accuracy of the data controls the iterative process. The degree of smoothing is dependent on a free smoothing parameter and on the accuracy of the data. The method is not restricted to special types of scattering functions. The termination error is negligible; therefore it is possible to correct parts of scattering distributions. This is of importance for investigations in biological substances where the scattering distribution must be determined in parts with different slit widths. No artificial oscillations are generated in the solution of the integral equation. To ensure the stability of the procedure a criterion for uniform convergence of the iterative process is involved. The results of several numerical tests are shown in the figures. The Fortran program was tested for several months with very satisfactory results.
\end{abstract}

\section{Introduction}

The correction of collimation errors in small-angle scattering is an absolute necessity (Guinier \& Fournet, 1955; Kratky, Porod \& Skala, 1960). The angular divergence and the wavelength distribution of the primary beam form the well known origin of the resolution errors (Hossfeld, 1968). They lead to an integral equation of first kind [equation (6)]. The scattered intensity is measured only in a limited angular range. This causes a termination effect which can never be completely removed. But it should be kept as small as pos- sible in order to make it negligible for practical use.

The scattering distributions are measured at a finite number of sampling points with statistical accuracy. If such a scattering distribution were corrected for resolution errors without prior smoothing, the statistical fluctuations would be amplified and would lead to strong oscillations of no physical significance.

One possible way of overcoming these difficulties is to produce a 'smooth' curve which fits the scattering data with a 'weighted least-squares' technique. To define the smoothness of a curve there must be a second mathematical condition. The approximated distribu- 